\title{
Clinical Results of Stenting for Cervical Internal Carotid Stenoses
}

\author{
M. TSUURA, T. TERADA, H. MATSUMOTO, O. MASUO, T. ITAKURA, G. HYOUTANI, \\ Y. NAKAMURA, T. NISHIGUCHI, H. MORIWAKI, S. HAYASHI
}

Department of Neurological Surgery Wakayama Medical University,

Department of Neurological Surgery Japanese Red Cross Society Wakayama Medical Center,

Department of Neurological Surgery Wakayama Rosai Hospital,

Department of Neurological Surgery Hashimoto Municipal Hospital,

Department of Neurological Surgery Hidaka General Hospital,

Department of Neurological Surgery Minami Wakayama National Hospital

Key words: stenting, internal carotid stenosis, protection device

\section{Summary}

Total 89 patients with cervical ICA stenosis were treated by stenting. In 74 cases of stenting, we used our blocking balloon systems to prevent distal embolism. The morbidity and the mortality rate was $4.5 \%$ and $0 \%$, respectively. Two $(3 \%)$ of 74 cases showed distal embolism when blocking balloon catheter systems(BBCS) were used, while distal embolism occurred in four $(27 \%)$ of 15 cases of stenting without BBCS. On diffusion-weighted MRI (DWI), hyperintense areas were detected in seven (47\%) of 15 lesions when we used BBCS only during postdilatation. On the other hand, use of $B B C S$ during predilatation as well as postdilatation reduced hyperintense areas on DWI, which were detected in three $(25 \%)$ of 12 patients. Our blocking balloon catheter system is a useful device to reduce the risk of distal embolism, especially when we use it during not only postdilatation but predilatation.

\section{Introduction}

Distal embolism is one of the serious complications of PTA/stenting for ICA stenosis, because cerebral embolism may result in severe neurological deficits or death. To prevent the distal embolism, we have used our original blocking balloon catheter systems (BBCS) in stenting for ICA stenosis. We report clinical experience of stenting for 89 patients with cervical ICA stenosis and our recent method where BBCS is used during predilatation as well as postdilatation

\section{Patients and Methods}

Stenting for cervical ICA stenoses was performed for 91 lesions of 89 patients between January 1997 and November 2001. In 74 patients (76 lesions), we used our blocking balloon systems in order to prevent distal embolism. In 37 cases (37 lesions), we utilized BBCS only during postdilatation after stent implantation, while BBCS was used during predilatation as well as postdilatation in late 38 cases (39 lesions).

Stent placement was performed under local anesthesia via percutaneous transfemoral route. Our blocking balloon catheter we usually use has a monorail type catheter tip on the silicone balloon and a 0.014" guidewire can pass the lumen of the tip. Briefly, we passed a 0.014 " microguidewire across the stenosis and then advanced the blocking balloon catheter through the stenosis along the microguidewire. After predilatation, a stent delivery system was navigated over the microguidewire and the stent was deployed.

When significant stenosis remained, postdi- 

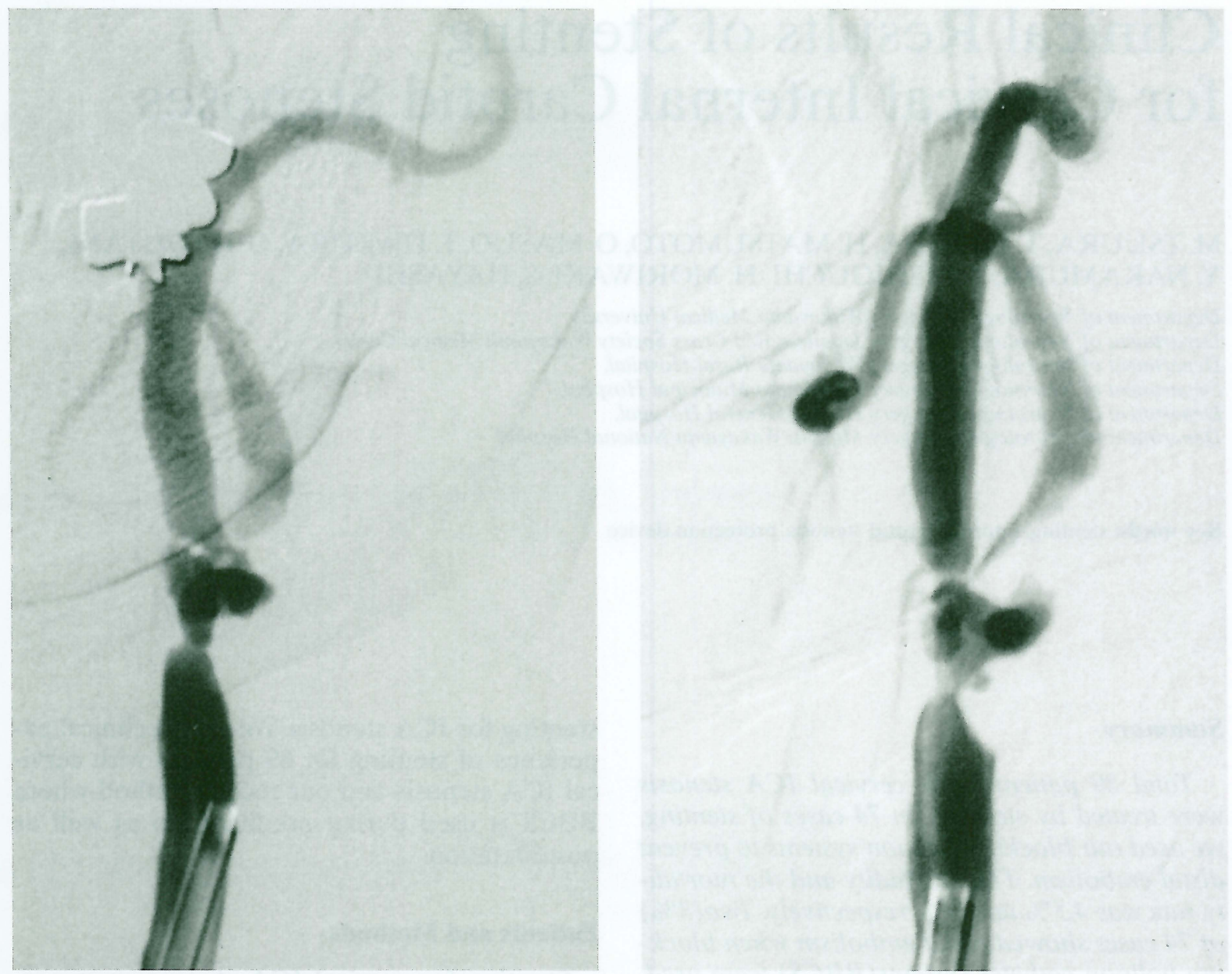

Figure 1 Angiogram before stenting for right severe ICA and CCA stenosis. Left and right picture shows AP view and lateral view of angiogram, respectively.

latation was performed under the use of BBCS. After postdilatation under flow arrest with BBCS, another 4 Fr catheter that had been placed in the common carotid artery before was advanced just proximal to the distal blocking balloon. Immediately blood in the ICA was aspirated and flushed into the external carotid artery through the 4 Fr catheter, then we deflated the distal blocking balloon and restored the blood flow. In early 37 cases (37 lesions), we used BBCS only during postdilatation described above.

However, in late 38 cases (39 lesions), we have used BBCS during predilatation as well as during postdilatation to reduce the hyperintensities on diffusion-weighted MRI after the procedure. Immediately after predilatation with $\mathrm{BBCS}$, the debris particles were washed into the external carotid artery with saline injected from the lumen of the PTA balloon catheter. We used SMART stents, Wallstents, Palmaz stents and Acculink in 59, 24, 7 and one patient, respectively. Angiography, conventional MRI (diffusion-weighted MRI in the possible cases) and CT scan were utilized to detect infarcts due to distal embolism.

\section{Results}

1. In 89 patients (91 lesions), the mean stenosis rate before and after stenting was $80.0 \%$ and $7.7 \%$, respectively. Morbidity was $4.5 \%$ (4 cases), which included 3 cases of TIA and one case of infarct due to distal embolism. There were $1.1 \%$ of neurological deficits at 30 days. Mortality was $0 \%$.

2. Restenosis occurred in two cases, at the rate of $3.0 \%$. Ipsilateral stroke rate was $1.1 \%$ 


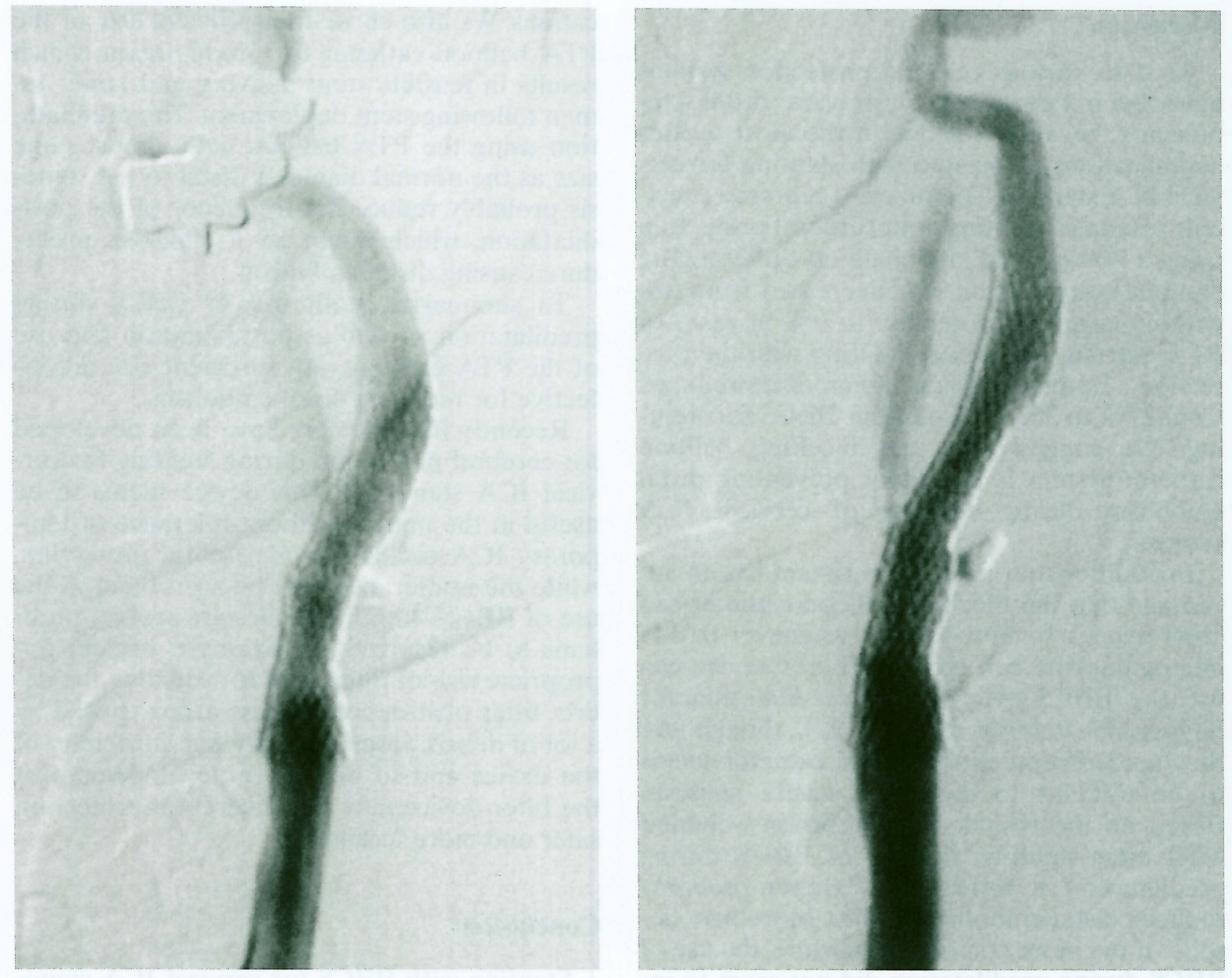

Figure 2 Angiogram after stenting for right severe ICA stenosis. Left and right picture shows AP view and lateral view of angiogram, respectively. Both BBCS during predilatation as well as postdilatation and the PTA balloon with sufficient size, are utilized in this case.

because one patient with the restenosis showed TIA. The patient was successfully treated by PTA.

3. Distal embolism occurred in two cases (3\%) of stenting with the BBCS and there was no neurological deficits at 30 days after stenting where BBCS were used. In contrast, distal embolism occurred in $4(27 \%)$ of 15 cases of stenting without BBCS and one patient showed neurological deficits at 30 days. Whether the BBCS was used or not, significantly related with distal embolism (Fisher's exact probability test: $\mathrm{p}=0.0062<0.05$ )

4. In 74 cases of stenting with BBCS, 30 days morbidity, permanent deficits and mortality was $1.4 \%, 0 \%$ and $0 \%$, respectively. In contrast, 30 days morbidity, permanent deficits and mortality was $20 \%, 6.7 \%$ and $0 \%$, respectively, in 15 cases of stenting without BBCS.
5. We performed DWI in 15 patients (15 lesions) after stenting using BBCS only during postdilatation (37 cases). Seven of 15 lesions (47\%) showed hyperintense areas on DWI. Therefore, to reduce the hyperintensities on DWI further, we began to use the BBCS routinely during predilatation (38 cases). In this new method, hyperintense areas were detected in three of 12 patients (25\%). In 37 cases of use of BBCS during postdilatation, 30 days morbidity, permanent deficits and mortality was $2.7 \%, 0 \%$ and $0 \%$, respectively. The mean stenosis rate before and after stenting was $77.8 \%$ and $9.9 \%$, respectively. In contrast, 30 days morbidity, permanent deficits and mortality was $0 \%$, respectively in 38 cases of use of BBCS during predilatation. The mean stenosis rate before and after stenting was $81.2 \%$ and $5.5 \%$, respectively. 


\section{Discussion}

To date, various cerebral protection systems have been developed to prevent distal embolism ${ }^{1-7}$, because it is one of the most serious complications associated with stenting for cervical ICA stenosis. Theron et Al reported excellent results of stenting with cerebral protection using a flow-guided latex balloon catheter. The embolic complication rate decreased from $8 \%$ without cerebral protection to $0 \%$ in cases of PTA and $2 \%$ in cases of stenting with the protection ${ }^{1}$. In this study, distal embolism reduced from $27 \%$ to $3 \%$ by use of our BBCS for stenting. We suggest that the blocking balloon catheter system is useful for preventing distal embolism during stenting of cervical ICA stenosis.

In addition, our protection system has an advantage that the blocking balloon catheter can cross even a severe stenosis, whenever 0.014" microguidewire can cross it. Therefore, we can use the BBCS even during predilatation for high-grade stenosis (figures 1,2), though the flow-guided protection balloon catheter seems to be difficult to cross the severe stenosis. Based on the results from diffusion-weighted MRI after stenting, the use of BBCS during predilatation as well as postdilatation probably reduced distal embolism further. Moreover, despite of the more complex procedure, the use of $\mathrm{BBCS}$ during predilatation did not seem to increase 30 days morbidity, permanent neurological deficits and mortality. Therefore we recently use our BBCS not only at postdilatation but also at predilatation to avoid embolic compli-

\section{References}

1 Theron JG, Payelle GG et Al: Carotid artery stenosis: treatment with protected balloon angioplasty and stent placement. Radiology 201: 627-636, 1996.

2 Albuquerque FC, Teitelbaum GP et Al: Balloon-protected carotid angioplasty. Neurosurgery 46: 918-923, 2000.

3 Martin JB, Pache JC et Al: Role of the distal balloon protection technique in the prevention of cerebral embolic events during carotid stent placement. Stroke 32: 479-484, 2001.

4 Al-Mubarak N, Roubin GS et Al: Effect of the distalballoon protection system on microembolization during carotid stenting. Circulation 104: 1999-2002, 2001.

5 Tubler T, Schluter M et Al: Balloon-protected carotid artery stenting: relationship of periprocedural neurological complications with the size of particulate debris. Circulation 104: 2791-2796, 2001. cations. We also chose the sufficient size of the PTA balloon catheter for predilatation, which results in feasible stent delivery and larger lumen following stent deployment. The predilatation using the PTA balloon with almost same size as the normal diameter distal to the stenosis probably reduce the frequency of the postdilatation, which might be a high-risk procedure causing distal embolism.

To summarize, both use of BBCS during predilatation as well as postdilatation and use of the PTA balloon with sufficient size are effective for reducing distal embolism.

Recently filter devices have been developed for cerebral protection during stenting for cervical ICA stenosis ${ }^{6,7}$. This device seems to be useful in the patient without tolerance of temporary ICA occlusion for cerebral protection, while the intolerance may be a problem in the use of BBCS. Although there are several problems to be resolved, for example, unclear appropriate size of filter pore for catching the debris, filter obstruction or flow arrest caused by a lot of debris, vascular injury due to rigidity of the device and so on, future developement of the filter device may make cerebral protection safer and more feasible.

\section{Conclusions}

Stenting with BBCS for cervical ICA stenosis is an effective treatment with low morbidity and mortality rate. Both predilatation and postdilatation using BBCS are useful techniques for reducing risk of distal embolism.

6 Reimers B, Corvaja N et Al: Cerebral protection with filter devices during carotid artery stenting. Circulation 104: 12-15, 2001

7 Angelini A, Reimers B et Al: Cerebral protection during carotid artery stenting: collection and histopathologic analysis of embolized debris. Stroke 33: 456-461, 2002.

Mitsuharu Tsuura, M.D.

Department of Neurological Surgery

Wakayama Medical University

811-1 Kimiidera, Wakayama City

Wakayama, 641-0012, Japan 